\section{Ranking programs for matched groups and combined distributions}

\section{ALFRED L. BROPHY \\ Behavioral Science Associates, West Chester, Pennsylvania}

McNally and Wood (1982) and Brophy (1983) have provided BASIC programs to rank scores in a single group, and Wood (1983) has extended McNally and Wood's program for use with matched groups. The present programs adapt Brophy's program, which is comparatively fast, for use with matched groups and with combined distributions formed by merging two or more independent groups. In addition, the programs include routines for calculation of some common nonparametric statistics.

Matched Groups. MATRANK is a BASIC program that sorts and ranks $N$ scores from each of $M$ matched groups. It is based on Brophy's (1983) SORTRANK program, but MATRANK has an improved method of processing tied scores, ${ }^{1}$ and it uses two-dimensional arrays, the second dimension of which contains the identification number of the group. MATRANK begins by prompting the user to enter $M, N$, and the individual scores. After sorting and ranking the data, MATRANK displays the scores in sorted order, with their subject numbers and ranks, for each group separately. Then the ranks are displayed in the original order of the scores for all groups together in an $\mathrm{N} \times \mathrm{M}$ matrix. The program next shows the sum and mean of the $M$ ranks for each of the $\mathrm{N}$ subjects.

MATRANK includes routines to calculate Spearman's rank correlation coefficient when $M=2$ and Kendall's coefficient of concordance $(W)$ and the associated $S$ statistic when $M>2$. The program calculates values from Kendall $(1975$, pp. 38,96$)$ to correct both correlation measures for tied ranks. MATRANK can easily be modified to perform Friedman's two-way analysis of variance of ranks with $\mathrm{N}$ treatments and $\mathrm{M}$ blocks. Alternatively, the approximate chi-square statistic (C) yielded by Friedman's test can be obtained by a simple transformation, $\mathrm{C}=$ $\mathrm{M}(\mathrm{N}-1) \mathrm{W}$.

Combined Distributions. ONERANK is a BASIC program that sorts and ranks scores in a single distribution that has been formed by the combination of $M$ groups of equal or unequal size. It is very similar to Brophy's (1983) SORTRANK program, but with the improved processing of ties used in MATRANK. ONERANK adds a special data-input routine, and it inserts the identification number of the group in the array formerly used for the subject identification number. The program is dimensioned to handle up to 100 subjects, but that can readily be changed, as explained in the program listing.

The author's mailing address is: Behavioral Science Associates, P.O. Box 748, West Chester, PA 19381.
After ranking the data, ONERANK displays the scores of the combined distribution in sorted order, together with the identification number of the group in which each score originated and the score's rank. The program then calculates and displays the sum and mean of the ranks for each of the $\mathbf{M}$ groups, as well as the number of runs in the combined distribution. ${ }^{2}$ Finally, ONERANK performs the Kruskal-Wallis test, and when $M=2$, Wilcoxon's ranksum test and the functionally equivalent Mann-Whitney $U$ test. The program makes corrections for tied ranks in the Kruskal-Wallis test and in the normal approximation to the statistic yielded by Wilcoxon's test.

Language. MATRANK and ONERANK are written in TRS-80 Level II BASIC, a version of Microsoft BASIC. The programs should run with little of no change under most other BASIC interpreters. If the version of BASIC used does not support DEFINT statements, those statements can be removed from the programs without affecting the results. The memory required by the programs varies with $M$ and $N$. With $M=4$ and $N=15$ in each group, either program runs in less than $3 \mathrm{~K}$.

Availability. A listing of the programs can be obtained without charge from Alfred L. Brophy, Behavioral Science Associates, P.O. Box 748, West Chester, PA 19381. For a TRS-80 Model I disk copy of the programs, enclose a system diskette and envelope with return postage.

\section{REFERENCES}

Brophy, A. L. (1983). A versatile sorting and ranking program. Behavior Research Methods \& Instrumentation, 15, 465-466.

Hull, C. H., \& NIE, N. H. (Eds.). (1981). SPSS update 7-9: New procedures and facilities for releases 7-9. New York: McGraw-Hill.

Kendall, M. (1975). Rank correlation methods (4th ed.). London: Griffin.

MCNALLY, K. A., \& WoOD, D. L. (1982). BASIC rank-order program. Behavior Research Methods \& Instrumentation, 14, 545.

WooD, D. L. (1983). Multidimensional sorting and matrix display. Behavior Research Methods \& Instrumentation, 15, 550.

\section{NOTES}

1. MATRANK improves SORTRANK's processing of ties in two ways. (l) The value of a counter variable in the routine no longer depends upon the exit value of the index of the preceding FOR-NEXT loop. This change is advisable because the exit value differs in different BASIC interpreters. (2) MATRANK uses a simplified method of calculating the mean rank (R) of a set of tied scores: $R=I+H / 2$, where $I$ is the original rank of the first tied score and $\mathrm{H}$ is the number of additional tied scores in the set.

2. When there are ties between scores from different groups, the number of runs varies, depending on the arbitrary order in which the tied scores are arranged. In such cases, the ties could be broken randomly, but it would be better to determine the number of runs under all possible orderings of the scores, or at least the maximum and minimum numbers of possible runs. The simple run-counting routine in ONERANK does not make these analyses. Even widely used programs may not count runs accurately when there are intergroup ties. For example, the Statistical Package for the Social Sciences (Hull \& Nie, 1981, p. 235) reports an incorrect result for the minimum number of runs in a relatively simple set of data.

(Revision accepted for publication August 17, 1984.) 